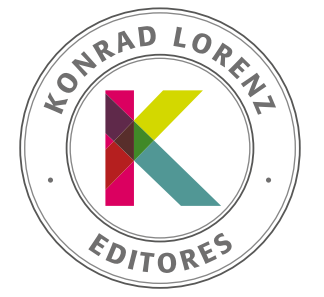

\title{
Extensão da história em FR e o desempenho subsequente em FI com custo
}

\author{
Talita Regina de Lima Cunhaa, Paula Renata Cordeiro de Lima ${ }^{b}$, \\ y Carlos Eduardo Costac,*
}

\author{
${ }^{a}$ Instituto de Psiquiatria do Hostpital da Clínicas da Faculdade de Medicina da USP (IPq-HCFMUSP)

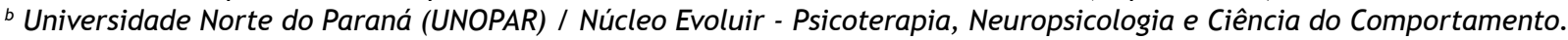 \\ ' Universidade Estadual de Londrina (UEL)
}

Received 7 August 2017; accepted 5 December 2017

\section{PALAVRAS-CHAVE história comportamental, extensão da história comportamental, persistência comportamental, custo da resposta, esquemas de reforçamento}

\section{KEYWORDS behavioral history, history extension, behavioral persistence, response cost, schedules of reinforcement}

\begin{abstract}
Resumo Alguns estudos indicam que taxas altas de respostas, produzidas por uma história de exposição ao esquema FR, tendem a persistir no esquema FI-custo. Todavia, esse resultado é controverso. Nos estudos em que foi observada a persistência de taxas altas, a extensão da história em FR era de $10 \mathrm{~h}$ e, naqueles em que não houve persistência, a extensão do FR foi de 45 min a $3 \mathrm{~h}$. 0 presente trabalho investigou o efeito da extensão da história de reforço sobre a persistência comportamental. Três grupos de participantes foram expostos a diferentes extensões de treino em FR ( 2 h 30 min, 5 h e 10 h), seguido por Fl-custo. Os resultados indicaram diminuição da taxa de respostas para a maioria dos participantes; quando houve persistência comportamental, ela não foi correlacionada com a extensão da história. (c) 2018 Fundación Universitaria Konrad Lorenz. Este es un artículo Open Access bajo la licencia CC BY-NC-ND (http://creativecommons.org/licenses/bync-nd/4.0/).
\end{abstract}

\section{History extension in FR and the subsequent performance in Fl-cost}

Abstract Some studies have indicated that high response rate, produced by a exposition history to a FR, persist in a FI-cost. However, this is a controversial result. In studies which high response rate was observed, the FR extension history was 10 hours and in those which no persistence was seen the duration of FR ranged from 45 minutes to 3 hours. The present paper investigated the effect of the extension of history of reinforcement on behavioral persistence. Three groups of participants were exposed to different training extensions in FR ( $2 \mathrm{~h} 30 \mathrm{~min} ; 5$ $\mathrm{h}$ e $10 \mathrm{~h}$ ), followed by an $\mathrm{Fl}$-cost. The results indicated a decrease on the response rate to most participants; when behavioral persistence happened was not correlated to history extension. (c) 2018 Fundación Universitaria Konrad Lorenz. This is an open access article under the CC BY-NCND license (http://creativecommons.org/licenses/bync-nd/4.0/).

* Autor para correspondencia.

E-mail do correo: caecosta@uel.br 
O comportamento de humanos e não-humanos sob esquemas de reforço é controlado pelas contingências presentes, mas também é afetado pela história de exposição a outros esquemas de reforço (e.g., Aló, 2005; Freeman \& Lattal, 1992; Lattal \& Neef, 1996; Wanchisen, 1990; Weiner, 1969, 1970). Uma questão importante e controversa em relação aos efeitos da história sobre o comportamento presente diz respeito à sua duração (Costa, Cirino, Cançado, \& Soares, 2009).

Em alguns estudos de história comportamental realizados por Weiner (e.g., Weiner, 1965, 1969, 1970) humanos que foram expostos a esquemas de razão (geralmente, mas não apenas, de razão-fixa - FR) mantiveram taxas de respostas relativamente mais altas em um esquema de intervalo-fixo (FI) subsequente do que aqueles que não tinham história de exposição a esquemas de razão ou haviam sido expostos a esquemas que selecionavam baixas taxas de respostas (e.g., reforço-diferencial-de-baixas-taxas - DRL). Esses resultados sugerem efeitos de longa duração da exposição ao FR sobre o desempenho de humanos em FI. Mesmo quando uma contingência de "custo da resposta" foi adicionada ao FI, após uma história de responder em FR, a persistência comportamental foi observada (Weiner, 1965, 1969, 1970). Nesses experimentos com custo, o cumprimento do esquema de reforço adicionava 100 pontos a um contador e o "custo da resposta" consistia na perda de um ponto para cada resposta emitida.

Todavia, a persistência de longa duração de altas taxas de respostas sob um FI-custo, após exposição a um esquema de reforço em FR não tem sido replicada (e.g., Costa, Soares, Becker \& Banaco, 2009; Costa, Souza, Bianchini, Porto, \& de Freitas; 2013). Costa, et al. (2009) expuseram 10 universitários aos seguintes esquemas de reforço, em sequência: FR 60, FI 15 s-custo 1, FR 60 e FI 15 s-custo 10. A cor do botão de respostas era uma nas condições de FR e outra nas condições de FI. Em todos os esquemas de reforço, quando a contingência era cumprida, 100 pontos eram creditados no contador; quando o custo da resposta estava programado cada resposta emitida antes do fim do intervalo subtraía um (custo 1) ou 10 (custo 10) pontos do contador de pontos. Cada esquema foi mantido por três sessões de 15 min cada. Oito dos 10 participantes diminuíram a taxa de respostas quando o esquema de reforço mudou de FR para FI-custo 1. Quando a contingência de FR foi posta em vigor novamente, as taxas de respostas foram mais altas que no Fl-custo 1 para todos os participantes e quando o esquema de reforço mudou novamente de FR para FI-custo 10 , a taxa de respostas diminuiu para todos os participantes. Costa et al. (2013) replicaram estes resultados utilizando um esquema múltiplo FR 60 FR 60 na linha de base e depois alterando o esquema para um múltiplo $\mathrm{Fl}$-custo $1 \mathrm{Fl}$-custo 10 na fase de teste, com controle da ordem de apresentação dos componentes entre os participantes. As taxas de respostas diminuíram para todos os participantes durante o teste, independentemente da ordem de apresentação dos componentes com custo 1 e custo 10 .

Tomados em conjunto, os resultados de Costa, et al. (2009) e Costa et al. (2013) não replicaram os resultados obtidos por Weiner $(1965,1969,1970)$. Replicação é fundamental para o avanço de qualquer ciência experimental, pois está intimamente relacionada a questão da generalidade dos resultados de qualquer pesquisa (Branch \& Pennypacker,
2013; Kantowitz, Roediger III, \& Elmes, 2006; Sidman, 1960). Um dos primeiros passos para a compreensão de por quê uma pesquisa não replicou os resultados de outro estudo semelhante é a avaliação sistemática das variáveis de procedimento. Assim, a extenção da história parece ser uma variável relevante a ser investigada. Nas pesquisas de Weiner (1965, 1969 e 1970) a exposição ao FR foi maior (i.e., $10 \mathrm{~h})$ que nos estudos de Costa, et al. (2009) e Costa et al. (2013) (i.e., 45 min e cerca de $3 \mathrm{~h}$, respectivamente). Lattal e Neef (1996) e Ayvasik, Fowler e Kallman (1996) sugeriram que pesquisas acerca da extensão da história são importantes e alguns estudos experimentais têm indicado que uma maior extensão da história pode estar relacionada com uma maior persistência comportamental (e.g., Siegel \& Foshee, 1953; Thompson, Heistad \& Palermo, 1963; Tombaugh \& Jean,1972; Traupmann \& Porter, 1971; Uhl, 1973).

Por exemplo, Uhl (1973) distribuiu 32 ratos em quatro grupos que foram expostos a um, três, nove ou 27 dias de treino em intervalo-variável (VI) $30 \mathrm{~s}$. Na primeira condição da fase de testes os ratos eram expostos a uma de duas condições: extinção ou um treino de omissão. No teste com treino de omissão, os ratos recebiam um reforço a cada $20 \mathrm{~s}$ se não pressionassem a barra e cada resposta adiava o reforço por $40 \mathrm{~s}$. Na segunda condição da fase de testes, todos os ratos eram expostos um esquema de tempo-variável (VT) 30 s com o objetivo de testar a "durabilidade" da eliminação da resposta. Os resultados indicaram que, tanto na extinção quanto na omissão (primeira fase de testes), a quantidade de tempo necessário para que o critério de supressão das taxas de respostas fosse atingido era diretamente proporcional à quantidade de treino. Os resultados também indicaram que a recuperação da pressão a barra sob o VT foi maior para os ratos que haviam passado por uma maior quantidade de treino e foram expostos a extinção na primeira condição da fase teste.

Por outro lado, Weiner (1982) não encontrou efeito da quantidade de treino sobre a taxa de respostas em uma fase de teste subsequente. Serão descritos aqui somente o Experimento 1 (Grupos 1 e 2) e o Experimento 5 (Grupo 24) por serem os únicos que têm relevância para o presente trabalho. Os participantes do Grupo 1 foram expostos somente a um FR por oito sessões; os participantes do Grupo 2 foram expostos a oito sessões sob um reforço-diferencial-de-outras-respostas (DRO), seguido pela exposição ao FR e os participantes do Grupo 24 foram expostos 16 sessões de DRO seguido pelo FR. Os resultados do Grupo 2 e do Grupo 24 indicaram que a exposição ao DRO gerou taxas de respostas mais baixas na fase teste em FR quando comparada com as taxas de respostas em FR dos participantes do Grupo 1 (sem exposição ao DRO). Não houve diferença nas taxas de respostas do Grupo 2 e do Grupo 24, ou seja, os efeitos supressivos da história em DRO sobre as taxas de respostas em FR não foram alterados pela extensão da história.

O experimento de Weiner (1982) difere daqueles outros citados anteriormente sobre os efeitos da extensão da história em alguns pontos. Weiner usou FR no teste, enquanto os outros experimentos utilizaram Extinção ou DRO e a maioria utilizou reforço em CRF na fase de história, exceto o de Uhl (1973). Ainda, o estudo de Kass e Wilson (1966), obteve resultados opostos (i.e., quanto maior foi a quantidade de treino menor foi a resistência a extinção). Crianças foram expostas a três, nove, 21, 45 ou 60 sessões de CRF (chamada 
de Condição 100\%) ou um esquema de reforçamento parcial (na qual ocorria liberação randômica de reforços, chamada de Condição 33,3\%). Os resultados sugeriram que, de modo geral a resistência à extinção foi inversamente proporcional à quantidade de treino para ambos os esquemas de reforço.

Tomados em conjunto, os estudos revisados para o presente trabalho sobre o efeito da quantidade de treino sobre a persistência comportamental, sugerem que o efeito pode ser de aumento na persistência, mas esse resultado pode depender de outras variáveis (e.g., os esquemas de reforço que são empregados na fase de história e teste; cf. Aló, Abreu-Rodrigues, Souza \& Cançado, 2015).

Em suma, uma vez que (a) os resultados da pesquisa de Weiner $(1965,1969,1970)$ não têm sido replicados por nenhum experimento conhecido até o momento (b) replicação é peça-chave de toda ciência experimental; (c) a extenção da história foi uma variável que diferiru entre os experimentos de Weiner e os de Costa, et al. (2009) e Costa et al. (2013), que buscaram realizar uma replicação dos resultados de Weiner, a presente pesquisa teve como objetivo principal avaliar se a persistência comportamental em FI-custo, após uma história de exposição a um FR, poderia ser influenciada pelo número de sessões na fase de história. 0 objetivo secundário foi avaliar se a persistência comportamental em FI-custo poderia estar relacionada com a estabilidade da taxa de respostas no FR.

\section{Método}

\section{Participantes}

Participaram 17 universitários (sete homens e 10 mulheres), entre 18 e 22 anos de idade (média de 20 anos), sem experiência com esquemas de reforço.

\section{Equipamentos, Instrumentos e Local}

A coleta de dados ocorreu em um laboratório de aproximadamente $27 \mathrm{~m}^{2}$, com quatro salas experimentais de aproximadamente $3 \mathrm{~m}^{2}$ cada. Dessas salas, duas foram utilizadas, cada uma equipada com uma mesa, uma cadeira, um ventilador, um computador, um fone de ouvido e uma filmadora digital montada sobre um tripé. Os computadores eram do tipo PC, com monitores de 14 polegadas em cores, teclado e mouse padrão. Para coleta de dados foi utilizado o software ProgRef v3.1 (Costa \& Banaco, 2002, 2003) e para o cálculo da estabilidade da taxa de respostas foi utilizado o software Stability Check (Costa \& Cançado, 2012).

\section{Procedimento}

Os materiais do participante (relógio, celular, livros, dentre outros) eram deixados no laboratório, fora da sala experimental. Antes do início da primeira sessão, os participantes liam o Termo de Consentimento Livre e Esclarecido (TCLE). De modo geral, o TCLE dizia que se tratava de uma pesquisa cujo objetivo era "estudar algumas variáveis que possam afetar o modo como as pessoas se comportam em determinadas situações"; informava ao participante o número e a duração das sessões experimentais; que se tratava de uma tarefa a ser executada no computador e que o objetivo era "ganhar o maior número de pontos possíveis (que aparecerão na tela do monitor) utilizando o mouse". Informava também que "cada 100 pontos presentes no contador serão trocados, ao final de cada sessão, por R\$ 0,03" e que o participante poderia abandonar a pesquisa a qualquer momento sem prejuízo.

Após assinarem o TCLE, os participantes recebiam a seguinte instrução, por escrito:

Esse trabalho não se trata de uma pesquisa sobre inteligência ou personalidade. Seu objetivo será ganhar pontos utilizando apenas o mouse. Os pontos aparecerão em uma janela (contador) que se localizará na parte superior da tela do computador, na posição central. $O$ experimentador não está autorizado a dar qualquer informação adicional. Caso haja dúvidas, releia estas instruções e prossiga o experimento. Bom trabalho!

$\mathrm{Na}$ primeira sessão, após a leitura, a folha de instrução era devolvida ao experimentador, era solicitado que o participante colocasse os fones de ouvido no qual soava um ruído branco, para efeito de isolamento acústico, a filmadora era ligada e, então, o experimentador saía da sala experimental. Nas demais sessões, quando o participante entrava na sala experimental a filmadora já estava ligada, o participante sentava-se, colocava o fone de ouvido e o experimentador saía da sala experimental.

Inicialmente, a tela do computador consistia em um fundo cinza claro (que era mantido inalterado ao longo de todas as sessões) e um retângulo (botão iniciar) cinza escuro na posição central superior com a palavra "Iniciar". Ao clicar com o cursor do mouse nesse botão, a sessão era iniciada com a apresentação de outra tela de fundo cinza, dessa vez com um retângulo no centro inferior (botão de respostas), um contador de pontos no centro superior (acima do botão de respostas) e um retângulo no canto superior direito (botão de resposta de consumação). Uma figura da tela da sessão pode ser vista em Costa e Banaco (2002).

A tarefa experimental consistia em clicar sobre o botão de respostas. Quando a exigência de um esquema de reforço era cumprida, aparecia no canto superior direito da tela (abaixo do botão de resposta de consumação) um ícone de um smile. 0 participante deveria então clicar com o cursor sobre o botão de resposta de consumação. Ao fazer isso, o smile desaparecia e 100 pontos eram adicionados ao contador.

Caso alguma contingência de reforço (FR ou Fl-custo) fosse cumprida no botão de respostas mais de uma vez, sem que o participante clicasse no botão de resposta de consumação, outro smile não aparecia (somente o que estava na tela continuava visível) e o participante não ganhava pontos enquanto não emitisse a resposta de consumação. Enquanto um smile estivesse visível na tela do computador o software continuava reiniciando a contagem do número de respostas a cada 40 respostas no $\mathrm{FR}$ e reiniciando o intervalo do $\mathrm{Fl}$ -custo depois da primeira resposta que ocorresse após $10 \mathrm{~s}$, e a subtração dos pontos (custo), quando programada, continuava a ocorrer normalmente.

Todos os participantes foram distribuídos, por sorteio, em três grupos que passaram por duas fases experimentais resumidas na Tabela 1. Foram designadas diferentes cores para o botão de respostas a depender do esquema de reforço em vigor (como nos estudos de Costa et al., 2009; 
Tabela 1 Resumo do procedimento experimental

\begin{tabular}{|c|c|c|c|c|c|c|c|}
\hline \multirow[b]{3}{*}{ Grupos } & \multirow[b]{3}{*}{$\mathrm{n}$} & \multicolumn{3}{|c|}{ Fase 1 - História } & \multicolumn{3}{|c|}{ Fase 2 - Teste } \\
\hline & & \multicolumn{3}{|c|}{ Botão Amarelo } & \multicolumn{3}{|c|}{ Botão Azul } \\
\hline & & Esquema & Sessões & Tempo $^{a}$ & Esquema & Sessões & Tempo $^{a}$ \\
\hline G1 & 6 & FR & 5 & $2 \mathrm{~h} 30 \mathrm{~min}$ & Fl-custo & 5 & $2 \mathrm{~h} 30 \mathrm{~min}$ \\
\hline G2 & 5 & FR & 10 & $5 \mathrm{~h}$ & Fl-custo & 5 & $2 \mathrm{~h} 30 \mathrm{~min}$ \\
\hline G3 & 6 & FR & 20 & $10 \mathrm{~h}$ & FI-custo & 5 & $2 \mathrm{~h} 30 \mathrm{~min}$ \\
\hline
\end{tabular}

Nota. FR = FR 40; FI-custo = FI 10 s-custo 1.

a Tempo total de exposição ao respectivo esquema de reforço (cada sessão durava 30 minutos).

Weiner, 1965, 1969, 1970).

Fase de História (FR). Os participantes de todos os grupos foram expostos a um esquema FR 40. Nesse esquema, um smile aparecia após 40 respostas emitidas desde o início da sessão e, a partir daí, desde o último cumprimento da razão; uma pressão no botão de resposta de consumação era seguida pelo desaparecimento do smile e pelo crédito de 100 pontos no contador. A cor do botão de respostas era amarela nesta fase. Os participantes do Grupo 1 eram expostos a cinco sessões; os participantes do Grupo 2, a 10 sessões; e os do Grupo 3, a 20 sessões, com duração de 30 min cada. Portanto, os participantes dos Grupos 1, 2 e 3 eram expostos ao FR por 2 h 30 min, 5 h e 10 h, respectivamente.

Fase de Teste (Fl-custo). Todos os participantes, dos três grupos, foram expostos a cinco sessões de 30 min cada, em um esquema FI $10 \mathrm{~s}$ com custo. No Fl-custo, cada resposta emitida após a passagem de $10 \mathrm{~s}$ tinha como consequência o aparecimento do smile e, após a resposta de consumação, 100 pontos eram creditados no contador. 0 intervalo do $\mathrm{FI}$ começava no início da sessão e, a partir daí, desde o cumprimento da exigência do último Fl. Portanto, o tempo gasto pelo participante para deslocar o cursor do mouse do botão de respostas até o botão de respostas de consumação e voltar para o botão de respostas era computado como parte do intervalo do FI. Cada resposta emitida no botão de respostas antes que o intervalo de $10 \mathrm{~s}$ tivesse terminado produzia a perda de um ponto, descontado diretamente do saldo total de pontos do contador. Se o marcador de pontos estivesse em zero, o contador ficava negativo (i.e., um sinal de menos “-” aparecia à esquerda do número no contador de pontos). A cor do botão de respostas era azul nesta fase.

Foram realizadas cinco sessões por semana, não mais do que duas por dia, com intervalo mínimo de 5 min entre elas.

Avaliação da estabilidade da taxa de respostas. Como o procedimento envolveu uma quantidade fixa de exposição à fase de construção da história, sem avaliação da estabilidade da taxa de respostas, era possível que quanto maior fosse a estabilidade da taxa de respostas na Fase de História (FR), maior seria a persistência comportamental quando a contingência se alterasse (cf. Joyce \& Chase, 1990). Para o cálculo da estabilidade das taxas de respostas foram consideradas as quatro últimas sessões de FR e foi utilizado o software Stability Check (Costa \& Cançado, 2012). O software calculou a diferença entre as médias das duas primeiras e das duas últimas sessões, e dividiu essa diferença pela média total das quatro sessões (cf. Schoenfeld, Cumming, \& Hearst, 1956). O resultado foi multiplicado por 100 , indicando o índice de estabilidade (IE). Quanto menor o valor do IE, maior a estabilidade das taxas de respostas.

\section{Resultados}

A Figura 1 exibe as taxas de respostas por minuto (R/min) para cada participante dos grupos 1, 2 e 3 nas fases de história (5, 10 e 20 sessões com FR, respectivamente) e de teste (FI-custo). Os participantes P4 (Grupo 1) e P15 (Grupo 3) abandonaram a pesquisa antes de cumprir as cinco sessões em $\mathrm{Fl}$-custo previstas no procedimento. Para facilitar a visualização dos resultados, em cada coluna da Figura 1 os gráficos foram ordenados, de cima para baixo, em função dos participantes que apresentaram comportamento mais persistente na fase de teste até os que apresentaram menor persistência.

Nas quatro últimas sessões em FR 40 os participantes do Grupo 1 emitiram taxas de respostas acima de 217 R/min; os do Grupo 2 emitiram taxas de respostas acima de 219 R/ min e os do Grupo 3 acima de 240 R/min. Quando a contingência mudou de FR para Fl-custo, notou-se variabilidade entre participantes no que diz respeito à manutenção ou alteração das taxas de respostas. Para a maioria dos participantes de cada grupo, a taxa de respostas diminuiu logo na primeira sessão (P3, P4, P5, P6 do Grupo 1; P8, P9 e P11 do Grupo 2; e P12, P14, P16 e P17 do Grupo 3) e para outros três participantes, na segunda (P7 do Grupo 2 e P13 do Grupo 3) ou terceira (P10 do Grupo 2) sessão. Apenas dois participantes do Grupo 1 (P1 e P2) e um participante do Grupo 3 (P15) não mostraram alterações na taxa de respostas com a implementação do Fl-custo.

O IE das quatro últimas sessões de FR de cada participante é apresentado na Figura 1. Os IE foram inferiores a $10 \%$ (com exceção do participante P1), sugerindo que a estabilidade da taxa de respostas, aparentemente, não teve relação com a persistência comportamental durante o FI-custo. Por exemplo, P2 (Grupo 1) e P15 (Grupo 3) continuaram apresentando taxas de respostas relativamente altas em Fl-custo, enquanto P3 (Grupo 1), P7 e P9 (Grupo 2) e P12 e P14 (Grupo 3) diminuíram na taxa de respostas, embora todos tenham apresentado valores de IE similares (entre $3,21$ e $4,49 \%)$.

Tomados em conjunto, os resultados sugerem que a persistência comportamental não esteve correlacionada com a extensão da história ou com a estabilidade da taxa de respostas durante a exposição ao FR. A maioria dos participantes diminuiu as taxas de respostas quando a contingência mudou (quatro de seis participantes do Grupo 1; os cinco participantes do Grupo 2 e cinco de seis participantes do Grupo 3, quando se compara a última sessão de FR com a última sessão de $\mathrm{Fl}$-custo).

A Figura 2 exibe os registros cumulativos, de todos os 
Grupo $1-2$ h 30 min FR

História Teste
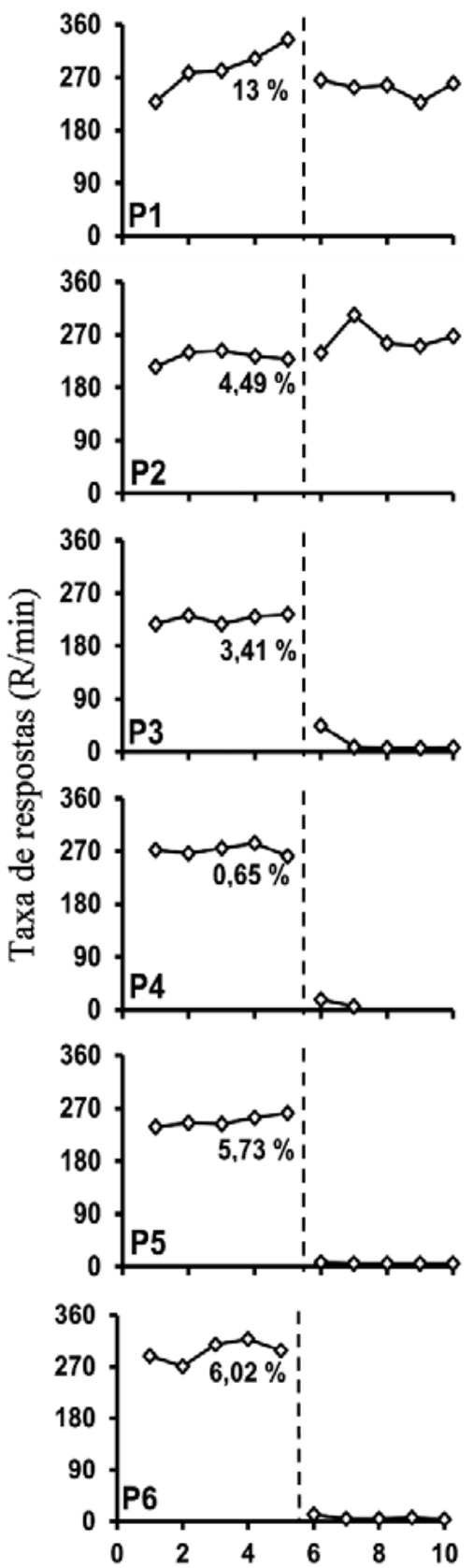

Grupo $2-5$ h FR

História
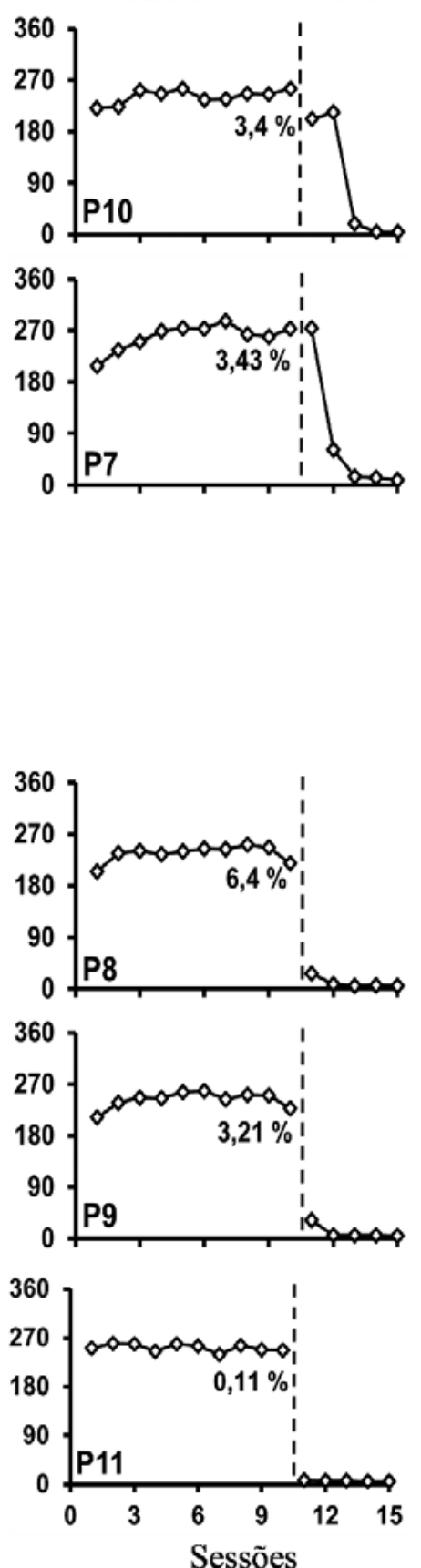

Grupo $3-10$ h FR

História Teste
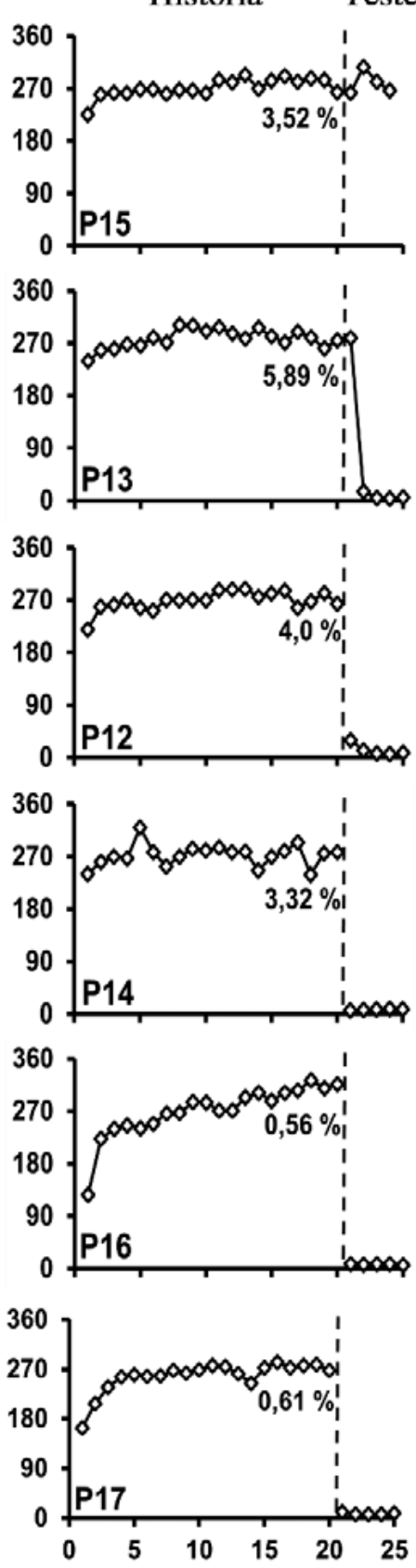

Figura 1 Taxa de respostas por sessão dos participantes do Grupo 1, Grupo 2 e Grupo 3. À esquerda da linha tracejada estão as sessões da Fase de História (FR) e à direita as da Fase de Teste (Fl-custo). Valores abaixo das últimas sessões de FR dizem respeito ao índice de estabilidade (IE).

participantes, das sessões de Fl-custo em que foi observada mudança de taxas de respostas altas para relativamente mais baixas pela primeira vez. Alguns participantes (P1, P2 e P15) não apresentaram mudanças substanciais nas taxas de respostas quando a contingência mudou de FR para FI-custo (ver Figura 1) e, nesses casos, foi selecionado o registro da última sessão em FI-custo. A letra $a$ refere-se à primeira transição de taxas de respostas altas para taxas de respostas relativamente mais baixas e a letra $b$ diz respeito ao aumento das taxas de respostas (bursts de respostas) quando um padrão de taxas de respostas relativamente mais baixo havia surgido anteriormente.

Foram identificados, nos registros cumulativos examinados, três padrões de transição de taxas de respostas altas para relativamente mais baixas durante FI-custo: (a) altas taxas de respostas (i.e., sem transição, quando nenhuma 
Grupo $1-2 \mathrm{~h} 30 \mathrm{~min}$ FR Grupo $2-5 \mathrm{~h}$ FR Grupo 3-10 h FR

P1 (5 $5^{\mathrm{a}}$ sessão de FI-Custo) $\quad$ P6 ( $\left(1^{\mathrm{a}}\right.$ sessão de FI-Custo $) \quad$ P12 $\left(1^{\mathrm{a}}\right.$ sessão de FI-Custo $)$

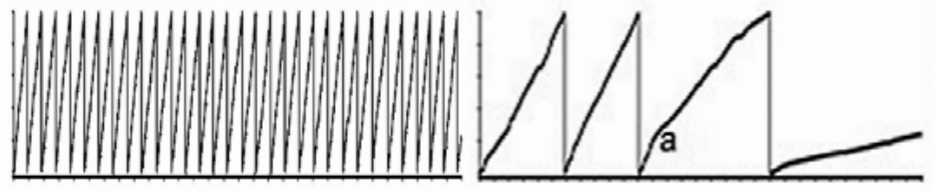

P2 (5 $5^{\mathrm{a}}$ sessão de FI-Custo)

P7 ( $2^{\mathrm{a}}$ sessão de FI-Custo)
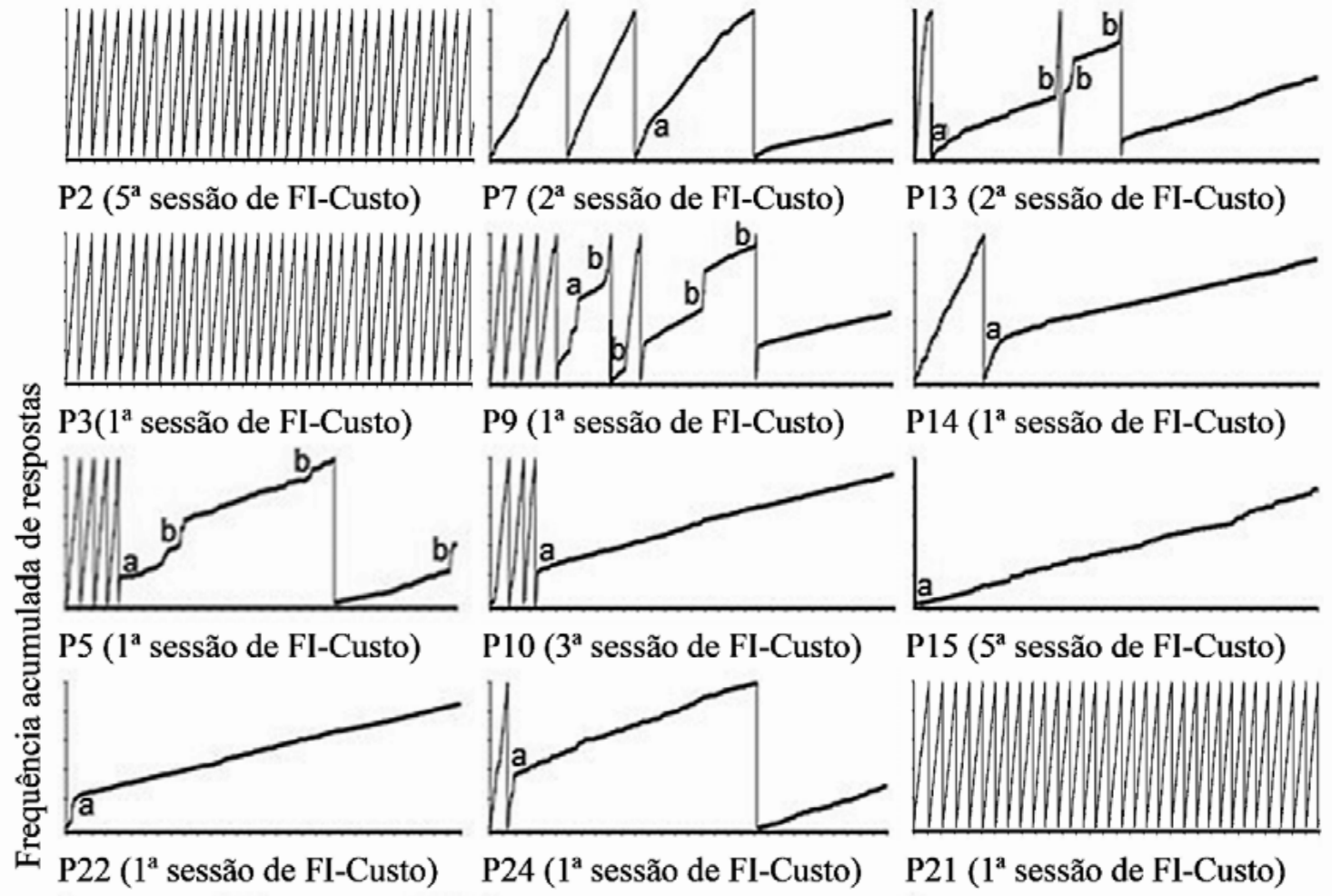

P9 ( $1^{\text {a }}$ sessão de FI-Custo)

P14 (1ª sessão de FI-Custo)
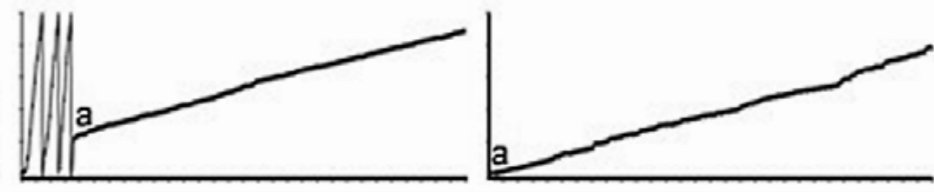

P10 ( $3^{\text {a }}$ sessão de FI-Custo)

P15 (5 sessão de FI-Custo)
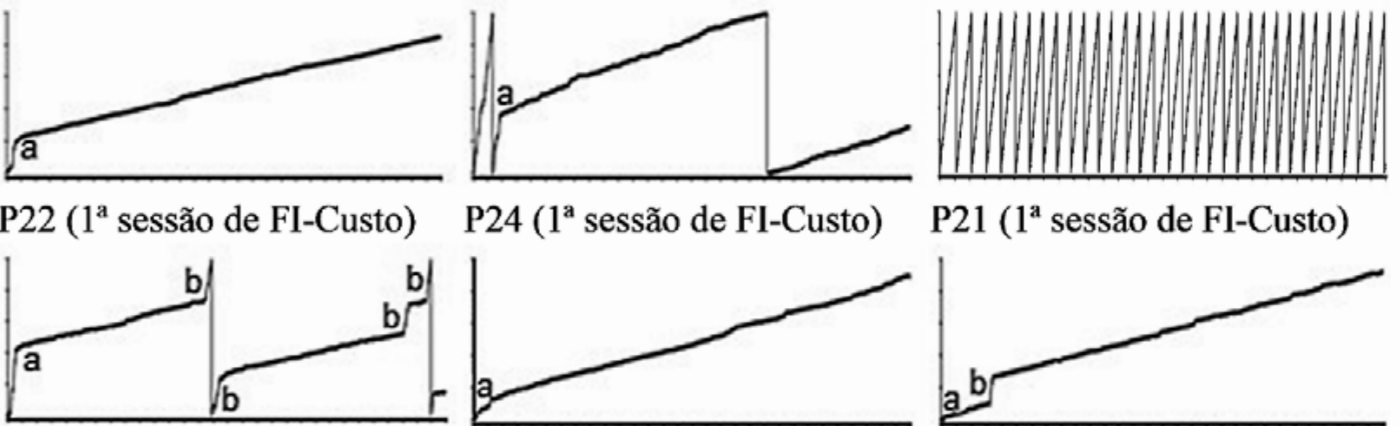

P24 ( $1^{\text {a }}$ sessão de FI-Custo)

P21 (1 $1^{\mathrm{a}}$ sessão de Fl-Custo)

P26 (1 $1^{\mathrm{a}}$ sessão de FI-Custo)
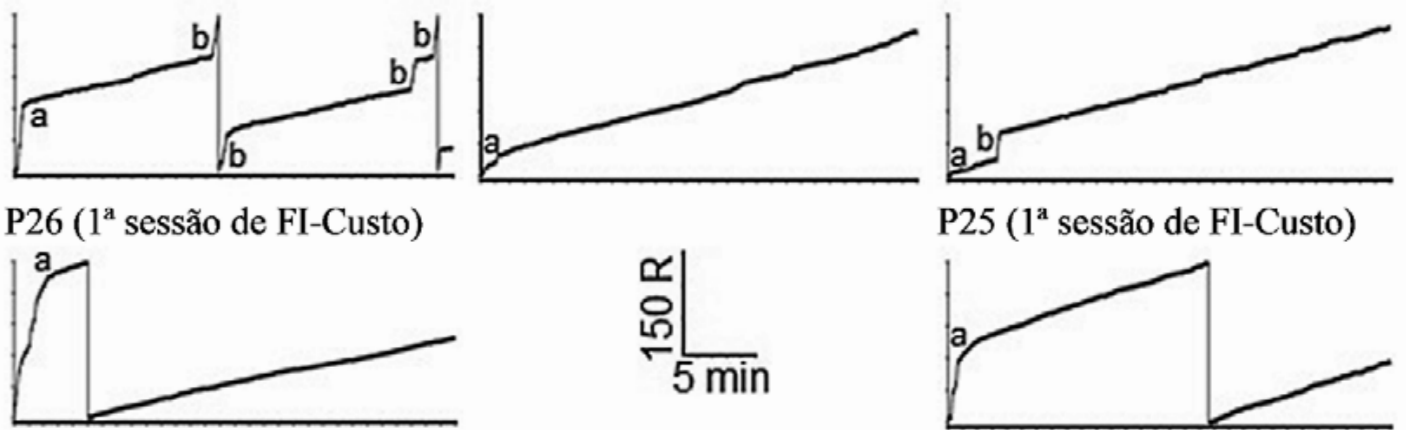

P25 ( $1^{\text {a }}$ sessão de FI-Custo)

Tempo (min)

Figura 2 Registros cumulativos das sessões de Fl-custo em que foi observada a mudança na taxa de respostas. A letra a refere-se à primeira transição de taxas de respostas altas para taxas de respostas relativamente mais baixas, e a letra b, ao aumento das taxas de respostas quando taxas de respostas baixas haviam surgido anteriormente. A curva do registro cumulativo retorna a zero após 250 respostas. Mais detalhes no texto.

letra está presente nos gráficos - P1 e P2 do Grupo 1; P15 do Grupo 3); (b) mudança de taxas de respostas alta para baixas e manutenção de taxas de respostas relativamente mais baixas (gráficos com apenas a letra a - P5 e P26 do Grupo 1; P6, P9, P10, P24 do Grupo 2; P13, P14 e P25 do Grupo 3) e (c) transição de taxas de respostas altas para relativamente mais baixas, com aumentos ocasionais da taxa no decorrer da sessão (gráficos com letras a e b - P3 e P22 do Grupo 1; P7 do Grupo 2; P12 e P21 do Grupo 3). Não houve relação sistemática entre esses padrões e a extensão da história, visto que houve incidência de dois ou três desses padrões para os participantes de todos os grupos do experimento. Portanto, quando a taxa de respostas mudou, a mudança foi em alguns casos relativamente "abrupta" (descritos pelo Padrão 2, acima) ou de maneira cíclica (descritos pelo $\mathrm{Pa}$ drão 3, acima).

A Figura 1 permite observar que a taxa de respostas diminuiu para a maioria dos participantes quando o esquema de reforço foi alterado, mas a Figura 3 permite avaliar se o comportamento dos participantes estava sob o controle do parâmetro temporal da contingência de FI. A Figura 3 exibe a porcentagem de respostas emitidas nos intervalos entre reforços (IRIs), em blocos sucessivos de 2 segundos, na última sessão da Fase de História (FR) e na primeira e última sessão da Fase de Teste (FI-custo). O intervalo 0,0-1,9 no eixo $x$ de cada gráfico exibe a porcentagem de respos- 
Grupo 1

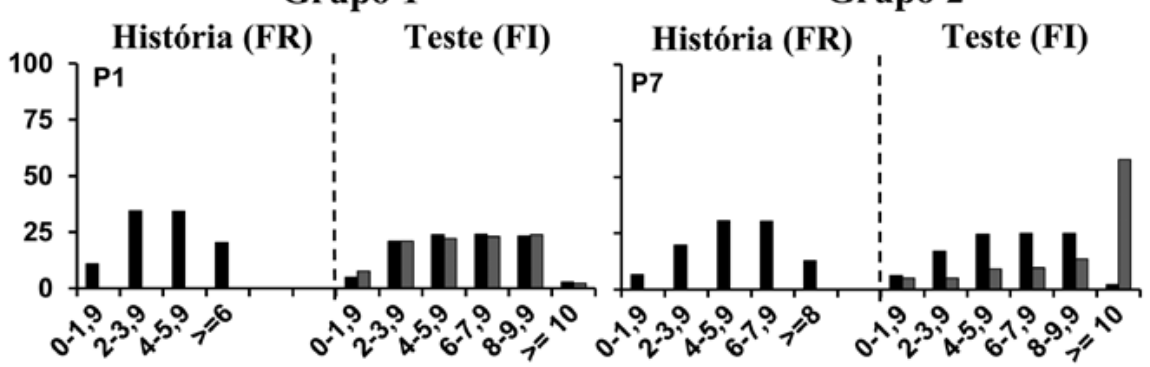

Grupo 2

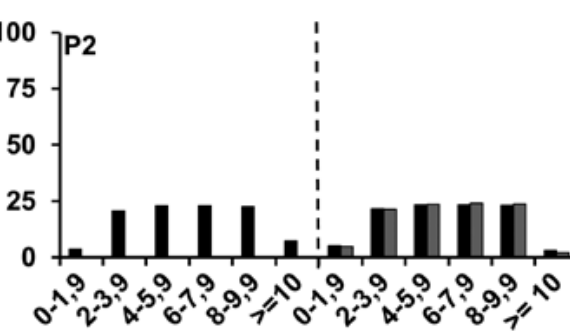

Grupo 3

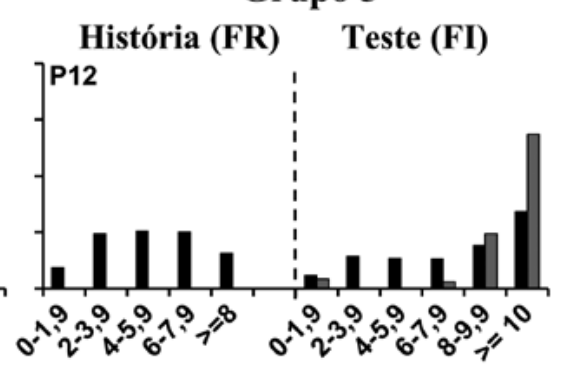

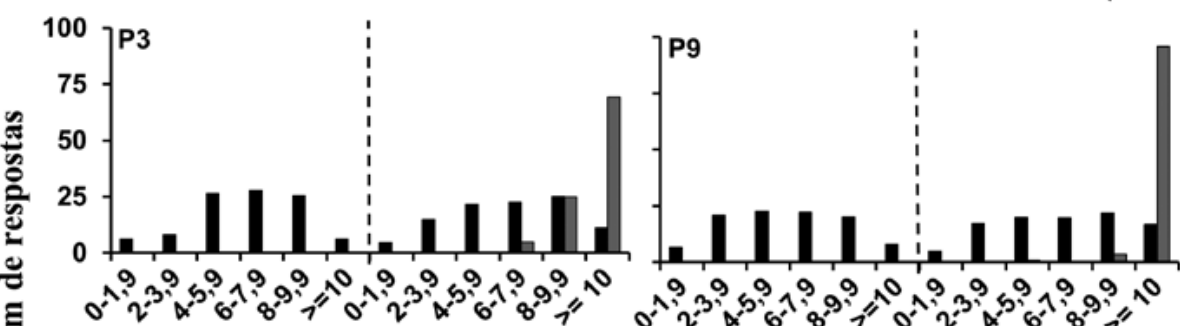

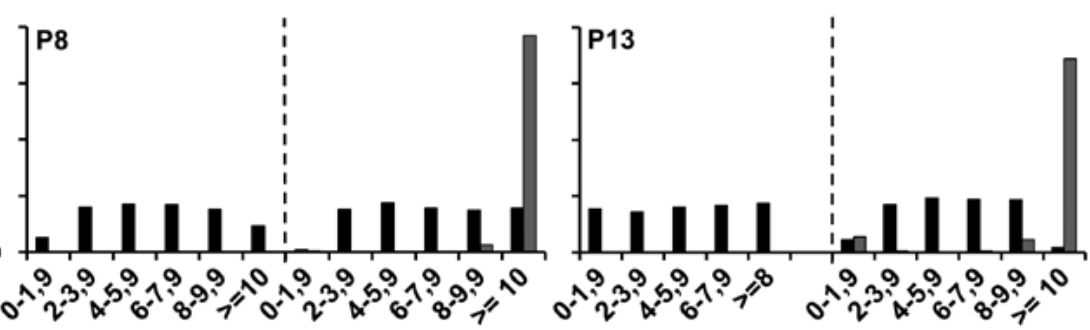

:

100
75
5
25

100

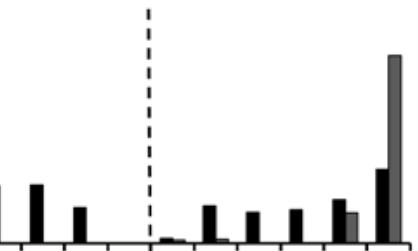

]P10

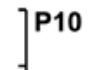

P14

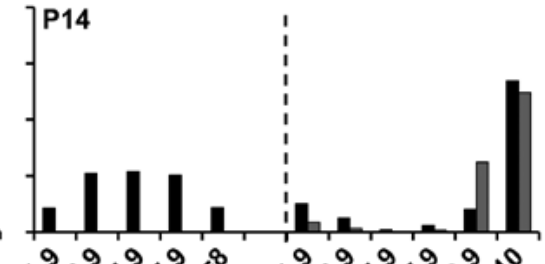

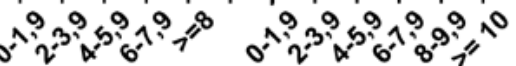
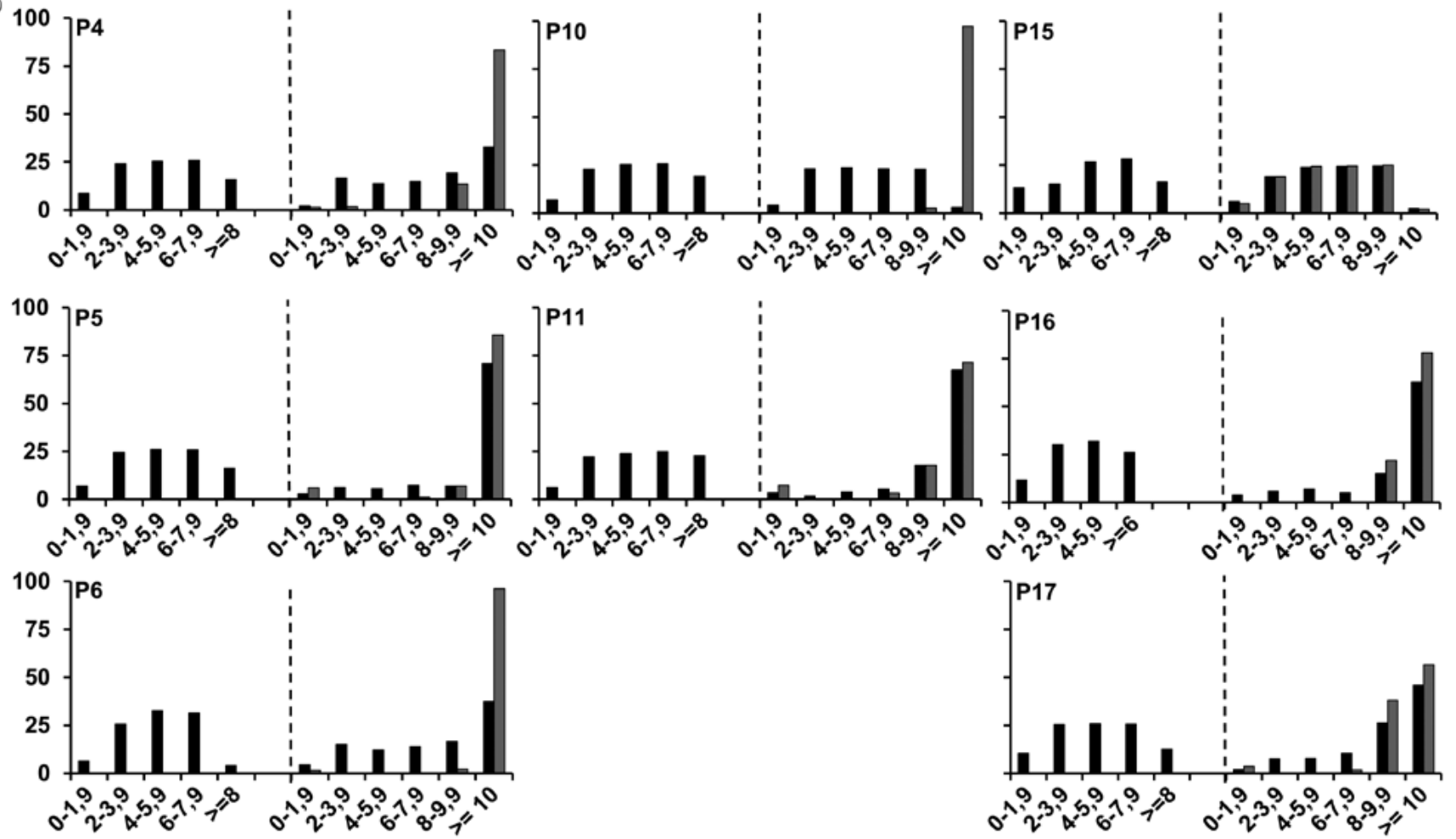

Blocos de 2 s

Figura 3 Porcentagem de respostas emitidas nos IRIs na última sessão da Fase de História (FR) e na primeira e última sessão da Fase de Teste (FI-custo), em blocos sucessivos de 2 segundos. Na Fase de Teste, as barras pretas referem-se à primeira sessão e as barras cinzas, à última sessão. Mais detalhes no texto. 
tas que foram emitidas dentro do intervalo de 1 décimo de segundo a 1,9 segundos 2 IRIs; o intervalo 2,0-3,9 exibe a porcentagem de respostas da sessão que foram emitidas dentro do intervalo de 2 a 3,9 segundos dos IRIs e assim por diante. Na Fase de Teste, as barras pretas referem-se à primeira sessão e as barras cinzas, à última sessão. Na fase de história, os blocos de intervalos foram construídos até o IRI médio de cada participante durante a exposição ao FR. Por exemplo, o IRI médio de P1 na última sessão de FR foi de $6 \mathrm{~s}$; por isso a distribuição da porcentagem de respostas no $F R$ vai até o bloco $\geq 6 \mathrm{~s}$. Este último bloco engloba porcentagem de respostas que foram emitidas no intervalo de $6 \mathrm{~s}$ ou mais. Quando o IRI médio foi de $7 \mathrm{~s}$ (P1 e P21) ou $9 \mathrm{~s}$ (P5; P22; P7; P10; P24; P12; P13; P15 e P25), o último bloco agrupou as respostas emitidas após $6 \mathrm{~s}$ ou $8 \mathrm{~s}$, respectivamente, para permitir que os blocos fossem sempre de $2 \mathrm{em}$ 2 segundos. Na fase de teste, uma vez que o valor do FI era $10 \mathrm{~s}$, o último bloco foi sempre $\geq 10 \mathrm{~s}$.

Observa-se na Figura 3 que, durante a exposição ao FR, uma porcentagem menor de respostas foi emitida no primeiro e último bloco de 2 s e a distribuição de respostas foi relativamente constante nos blocos intermediários. A menor porcentagem de respostas emitidas no primeiro bloco de 2 s deve-se, principalmente, ao tempo gasto para a emissão da resposta de consumação e, no último bloco, às eventuais emissões das 40 respostas requeridas pelo FR em um intervalo menor do que a média do IRI. É possível avaliar que a distribuição das respostas durante o IRI no FR foi relativamente constante, indicando que a taxa de respostas tendeu a ser constante nos IRIs.

Durante a exposição ao Fl-custo, é possível observar três padrões na distribuição das respostas durante o IRI: (a) um padrão semelhante ao da Fase 1 tanto na primeira quanto na última sessão do Fl-custo (i.e., persistência comportamental) para P1 e P2 (Grupo 1) e P15 (Grupo 3). Esses são os participantes que mantiveram altas taxas de respostas após a mudança da contingência de reforço (ver Figura 1); (b) um padrão semelhante ao da Fase 1 na primeira sessão da Fase 2 (barras pretas), mas uma alteração da distribuição das respostas na última sessão da Fase 2 (barras cinzas), isto é, a maioria das respostas passou a ser emitida no final do intervalo do FI (P3 do Grupo 1; P6, P7 P9 e P10 do Grupo 2; P13 do Grupo 3); (c) uma alteração da distribuição das respostas logo na primeira sessão da Fase 2 (i.e., a maior parte das respostas passaram a ser emitidas no final do intervalo do $\mathrm{FI}$ ), que se manteve (ou acentuou-se) na última sessão (P5, P22 e P26 do Grupo 1; P24 do Grupo 2; P12, P14, P21 e P25 do Grupo 3).

Tomados em conjunto, esses resultados sugerem que a taxa de respostas não apenas diminuiu com a mudança do FR para o Fl-custo para 14 dos 17 participantes, mas que a distribuição das respostas no IRI também mudou, com maior taxa de respostas sendo emitidas no final do intervalo, sugerindo que o comportamento desses participantes estava sob o controle do parâmetro temporal do FI.

\section{Discussão}

O objetivo da presente pesquisa foi avaliar se a persistência comportamental em Fl-custo, após uma história de exposição a um FR, poderia ser influenciada pela extensão da história. Os resultados do presente estudo indicaram que não houve persistência comportamental, como aquelas observadas nos estudos de Weiner $(1965,1969,1970)$ para a maioria dos participantes. Sugere-se que as contingências atuais de Fl exerceram maior influência sobre a taxa de respostas na fase de teste que as contingências passadas (cf. Freeman \& Lattal, 1992; Okouchi, 2003). Portanto, os resultados da presente pesquisa não dão suporte a afirmação de Weiner (1982) de que os desempenhos observados sob esquemas de reforço são, frequentemente, "mais uma função da história de reforço do que de contingências de reforço correntes" (p.489).

No presente estudo, quando houve persistência de taxas de respostas mais altas, selecionadas na fase de história em FR, essa persistência não foi correlacionada com a extensão da história, corroborando outros estudos que também não encontraram relação sistemática entre a extensão da história e a persistência comportamental com humanos (e.g., Kass \& Wilson, 1966; Lewis \& Duncan, 1958) e não-humanos (e.g., Ison, 1962; Senkowski, 1978). Os resultados do presente estudo também corroboram o de outras pesquisas nasquais houve sensibilidade à mudança nas contingências sobre a taxa de respostas com humanos (e.g., Costa, et al., 2009; Costa et al., 2013, Okouchi, 2003), até mesmo quando o comportamento estava sob o controle instrucional (e.g., Baumann, Abreu-Rodrigues, \& Souza, 2009; Rosenfarb, Newland, Brannon, \& Howey, 1992; Otto, Torgrud, \& Holborn, 1999).

Estudos que obtiveram uma relação direta entre quantidade de treino e persistência comportamental usaram CRF como a contingência na fase de história e extinção no teste (e.g., Thompson, Heistad, \& Palermo,1963; Traupmann \& Porter, 1971; Tombaugh \& St. Jean, 1972) ou VI na história e CRF ou treino de omissão no teste (Uhl, 1973). Os resultados de Aló et al. (2015) sugerem que os efeitos da história de reforço podem ser função do esquema de reforço que se utiliza no teste. Aló et al. realizaram três experimentos que investigaram os efeitos de histórias de FR e DRL sobre a persistência comportamental com pombos. Após a linha de base (LB) em um múltiplo FR DRL com taxas de reforços similares entre os componentes, alimentação prévia foi utilizada como disruption operation. A fase teste delineada, poderia ser (a) sob o mesmo esquema de reforço da LB (Experimento 1); (b) sob um esquema múltiplo EXT EXT (Experimento 2) e (c) sob um esquema múltiplo FI FI (Experimento 3). A taxa de respostas em FR foi menos persistente do que aquela em DRL (Experimentos 1 e 2), mas o resultado oposto foi verificado no Experimento 3, o que sugere que a maior persistência do comportamento selecionado por um esquema de reforço de baixas taxas (e.g., DRL) pode ser função, entre outras coisas, do esquema de reforço que se utiliza no teste.

O resultado de Aló et al. (2015) pode explicar a divergência entre os experimentos que utilizaram EXT (ou treino de omissão) no teste e os do presente experimento que utilizou FI na fase de teste e os de Weiner (1982) que utilizou FR no 
teste. Porém, não explica a divergência dos resultados do presente estudo, os de Costa, et al. (2009) e Costa et al. (2011) com os de Weiner $(1965,1969,1970)$, pois estes experimentos utilizaram FR na LB e FI-custo no teste.

A divergência entre os resultados aqui obtidos e os de Weiner (1965, 1969, 1970) é devida a outras variáveis que não a extensão da história. Existem ainda características do procedimento experimental de Weiner, que diferem do procedimento utilizado no presente estudo, que ainda não foram investigadas sistematicamente. Uma possibilidade seria a presença vs. ausência da resposta de consumação. Costa, Patsko e Becker (2007), Matthews, Shimoff, Catania e Sagvolden (1977) e Raia, Shillingford, Miller Jr. e Baier (2000) obtiveram resultados que sugerem a importância da resposta de consumação em, por exemplo, gerar pausas pós-reforço e, muitas vezes, dar origem à queda nas taxas de respostas em esquemas de intervalos (FI ou $\mathrm{VI}$ ), quando comparadas com as taxas de respostas na ausência de uma resposta de consumação. Portanto, a ausência da resposta de consumação nos estudos de Weiner pode ter contribuído para a maior persistência comportamental do que a observada no presente estudo e nos de Costa, et al. (2009) e Costa et al. (2013), que usaram uma resposta de consumação.

Experimentos na área de comportamento governado por regras têm sugerido que o tipo de instrução empregada (e.g., instruções mínimas vs. completas) podem tornar o comportamento mais ou menos sensível à mudança nas contingências de reforço (e.g., Albuquerque \& Ferreira 2001; Costa, Calixto, \& Banaco, 2017; Hayes, Brownstein, Haas, \& Greenway, 1986). A instrução de Weiner (1969) era mais completa do que a do presente estudo e isso pode ter contribuído para a diferença nos resultados.

Todavia, Weiner (1965) e Costa et al. (2013) instruíram os participantes a ganhar o maior número de pontos possíveis (mas não diziam nada sobre a perda de pontos) e os resultados desses dois estudos foram divergentes (apesar das instruções semelhantes). Weiner $(1969,1970)$ e Costa et al. (2009) instruíram os participantes a ganhar o maior número de pontos possíveis e informavam que eles poderiam perder pontos no decorrer do experimento e os resultados desses estudos (apesar da semelhança na instrução) também foram divergentes. Portanto, informar aos participantes que eles devem maximizar o ganho de pontos e/ou informar sobre a possibilidade de perda de pontos não parece ter influenciado na maior ou menor persistência (ou sensibilidade) comportamental quando a contingência mudou de FR para Fl-custo. A replicação direta do estudo de Weiner inclusive com a mesma instrução utilizada em seus estudos precisa ser levada à cabo.

Altas taxas de respostas nas primeiras sessões de Fl-custo e a baixa emissão de respostas, seguida por aumentos ocasionais nesse mesmo programa (ver Figura 2 - com letras $a$ e $b$, respectivamente), permitem sugerir que o custo estabelecido no presente estudo (perda de um ponto) não levou a uma queda abrupta na taxa de respostas. Essa mudança gradual na taxa de respostas, que ocorreu intra sessão (exceto para P14), permite especular que não houve comunicação entre a maioria dos participantes quanto a mudança na contingência de reforço, mas que as novas contingências (i.e., mudança no programa de reforço de FR para FI e a inclusão da perda de pontos) selecionou um novo padrão comportamental (i.e., mudança na taxa de respostas e na distribuição destas respostas).

A manutenção das altas taxas de respostas obtidas no Fl-custo por Weiner $(1965,1969,1970)$ poderia ser relacionada aos padrões eventualmente mais estáveis das taxas de respostas obtidas na fase de história em FR (cf. Joyce \& Chase, 1990) - embora Weiner não tenha adotado um critério de estabilidade, nem a tenha avaliado. Análise visual da estabilidade da taxa de respostas do estudo de Weiner (1969, Experimento 2, Figuras 5 e 7) sugerem, mas não garantem, que a estabilidade das quatro últimas sessões em FR eram comparáveis com a estabilidade encontrada para a maioria dos participantes do presente estudo (Figura 1), mas, mesmo assim, os resultados foram divergentes. Costa et al. (2013) também não encontrou relação entre estabilidade da taxa de respostas na fase de construção de história e a persistência comportamental.

Tomados em conjunto, os resultados obtidos no presente estudo sugerem que, em geral, quando uma contingência de reforço em FR muda para FI-custo, as taxas de respostas relativamente altas tendem a diminuir; a distribuição das respostas no tempo tende a se alterar e essas mudanças independem da extensão da história de responder em FR. Esses resultados corroboram aqueles obtidos nos estudos de Costa, et al. (2009) e Costa et al. (2013), mas não com os de Weiner (1965, 1969, 1970). Além da extensão da história, o tipo de instrução fornecida, o tipo de reforçador empregado, a estabilidade da taxa de resposta na fase de construção da história ou mesmo semelhanças ou diferenças (acidentais) no IRI entre as fases de história e de teste, não parecem explicar a diferença nos resultados encontrados entre os estudos. Uma diferença no procedimento que ainda não foi diretamente investigada é o emprego de uma resposta de consumação para cada reforço liberado ou mesmo uma replicação mais direta possível dos estudos de Weiner.

\section{Financiamento}

Esta pesquisa contou com o apoio financeiro da Fundação Araucária do Paraná (Programa de Pesquisa Básica e Aplicada - Chamada: 14/2009, Convênio UEL: 07/2011, Protocolo: 10924).

\section{Referências}

Albuquerque, L. C. , \& Ferreira, K. V. D. (2001). Efeitos de regras com diferentes extensões sobre o comportamento humano. Psicologia: Reflexão e Crítica, 14(1), 143-155. http://dx.doi. org/10.1590/S0102-79722001000100012

Aló, R. M. (2005). História de reforçamento. In J. Abreu-Rodrigues \& M. R. Ribeiro (Org.). Análise do Comportamento: pesquisa, teoria e aplicação (pp. 45-62). Porto Alegre, RS: Artmed.

Aló, R. M., Abreu-Rodrigues, J., Souza, A. S., \& Cançado, C. R. $X$. (2015). The persistence of fixed-ratio and differential-reinforcement-of-low-rate schedule performances. Revista Mexicana de Análisis de la Conducta, 41(1), 3-31.

Ayvasik, H. B., Fowler, S. C., \& Kallman, M. J. (1996). Midazolam's dose effects on fixed-ratio response rate in rats depend on amount of prior training experience. Experimental and Clinical Psychopharmacology, 4(2), 151-156. http://dx.doi. org/10.1037/1064-1297.4.2.151

Baumann, A. A. L., Abreu-Rodrigues, J., \& Souza, A. S. (2009). Rules and self rules: Effects of variation upon behavioral sensitivity to change. Psychological Record, 59, 641-670. http:// 
dx.doi.org/10.1007/BF03395685

Branch, M. N., \& Pennypacker, H. S. (2013). Generality and generalization of research findings. In G. J. Madden (Ed.), APA Handbook of Behavior Analysis: Vol. 1. Methods and Principles (Vol. 1, pp. 151-175). Washington, DC: American Psychological Association. http://dx.doi.org/10.1037/13937-007

Costa, C. E., \& Banaco, R. A. (2002). ProgRef v3: sistema computadorizado para coleta de dados sobre programas de reforço com humanos - recursos básicos. Revista Brasileira de Terapia Comportamental e Cognitiva, 4(2), 173-192.

Costa, C. E., \& Banaco, R. A. (2003). ProgRef v3: sistema computadorizado para coleta de dados sobre programas de reforço com humanos - recursos adicionais. Revista Brasileira de Terapia Comportamental e Cognitiva, 5(2), 219-229.

Costa, C. E., Calixto, F. C., \& Banaco, R. A. (2017). O efeito de instruções e de mudanças de instruções sobre o comportamento em DRL. Temas em Psicologia, 25(1), 81-96. http://dx.doi. org/10.9788/tp2017.1-05

Costa, C. E., \& Cançado, C. R. X. (2012). Stability check: A program for calculating the stability of behavior. Mexican Journal of Behavior Analysis, 38(1), 61-71.

Costa, C. E., Cirino, S. D., Cançado, C. R. X., \& Soares, P. G. (2009). Polêmicas sobre história comportamental: identificação de seus efeitos e sua duração. Psicologia. Reflexão e Crítica, 22(3), 294403. http://dx.doi.org/10.1590/S0102-79722009000300010

Costa, C. E., Lacerda, R. F. F., Paulino, L. R. P., \& Banaco, R. A. (2014). Efeito de uma história de FR sobre a taxa de respostas em FI e DRL. Perspectivas em Análise do Comportamento, 5(1), 1-16.

Costa, C. E., Patsko, C. H., \& Becker, R. M. (2007). Desempenho em FI com humanos: efeito da interação da resposta de consumação e do tipo de instrução. Interação em Psicologia, 11(2), 175-186. http://dx.doi.org/10.5380/psi.v11i2.11609

Costa, C. E., Soares, P. G., Becker, R. M., \& Banaco, R. A. (2009). $O$ efeito da magnitude do custo da resposta e do evento consequente empregado sobre o comportamento em FI após uma história de FR. Revista Brasileira de Análise do Comportamento, 5(2), 89-106.http://dx.doi.org/10.18542/rebac.v5i2.932

Costa, C. E., Souza, J. P. P., Bianchini, T., Porto, T. H., \& Freitas, L. A. B. (2013). Diferentes magnitudes do custo da resposta em um múltiplo $\mathrm{FI} \mathrm{FI,} \mathrm{após} \mathrm{uma} \mathrm{história} \mathrm{em} \mathrm{um} \mathrm{múltiplo} \mathrm{FR} \mathrm{FR.} \mathrm{In-}$ teração em Psicologia, 17(1), 11-26. doi:10.5380/psi.v17i1.26659

Freeman, T. J., \& Lattal, K. A. (1992). Stimulus control of behavioral history. Journal of the Experimental Analysis of Behavior, 57(1), 5-15. http://dx.doi.org/10.1901/jeab.1992.57-5

Hayes, S. C., Brownstein, A. J., Haas, J. R., \& Greenway, D. E. (1986). Instructions, multiple schedules, and extinction: Distinguishing rule-governed from schedule-controlled behavior. Journal of the Experimental Analysis of Behavior, 46(2), 137147. doi:10.1901/jeab.1986.46-137

Ison, J. R. (1962). Experimental extinction as a function of number of reinforcements. Journal of Experimental Psychology, 64(3), 314-317. http://dx.doi.org/10.2466/pr0.1964.14.2.647

Joyce, J. H., \& Chase, P. N. (1990). Effects of response variability on the sensitivity of rule-governed behavior. Journal of the Experimental Analysis of Behavior, 54(3), 251-262. http://dx.doi. org/10.1901/jeab.1990.54-251

Kantowitz, B., Roediger III, H., \& Elmes, D. (2006). Psicologia experimental: psicologia para compreender a pesquisa em psicologia. (R. Galman, G. Y. Tomanari, \& L. S. Barba, Trans. $8^{a}$ ed.). São Paulo, SP: Thomson Learning Edições.

Kass, N., \& Wilson, H. (1966). Resistance to extinction as a function of percentage of reinforcement number of training trials, and conditioned reinforcement. Journal of Experimental Pschology, 71(3), 355-357. http://dx.doi.org/10.3758/BF03209277

Lattal, K. A., \& Neef, N. A. (1996). Recent reinforcement-schedule research and applied behavior analysis. Journal of Applied Behavior Analysis, 29(2), 213-230. doi:10.1901/jaba.1996.29-213

Lewis, D. J., \& Duncan, C. P. (1958). Expectation and resistance to extinction of a lever-pulling response as a function of percentage of reinforcement and number of acquisition trials. Jour- nal of Experimental Psychology, 55(2), 121-128. http://dx.doi. org/10.1037/h0046622

Matthews, B. A., Shimoff, E., Catania, A. C., \& Sagvolden, T. (1977). Uninstructed human responding: Sensitivity to ratio and interval contingencies. Journal of the Experimental Analysis of Behavior, 27(3), 453-467. http://dx.doi.org/10.1901/ jeab.1977.27-453

Okouchi, H. (2003). Effects of differences in interreinforcer intervals between past and current schedules on fixed-interval responding. Journal of the Experimental Analysis of Behavior, 79(1), 49-64. http://dx.doi.org/10.1901/jeab.2003.79-49

Otto, T. L., Torgrud, L. J., \& Holborn, S. W. (1999). An operant blocking interpretation of instructed insensitivity to schedule contingencies. The Psychological Record, 49, 663-684. http:// dx.doi.org/10.1007/BF03395334

Raia, C. P., Shillingford, S. W., Miller Jr., H. L., \& Baier, P. S. (2000). Interaction of procedural factors in human performance on yoked schedules. Journal of the Experimental Analysis of Behavior, 74(3), 265-281. http://dx.doi.org/10.1901/jeab.2000.74265

Rosenfarb, I. S., Newland, M. C., Brannon, S. E., \& Howey, D. S. (1992). Effects of self-generated rules on the development of schedule-controlled behavior. Journal of the Experimental Analysis of Behavior, 58, 107-121. doi:10.1901/jeab.1992.58- 107

Senkowski, P. C. (1978). Variables affecting the overtraining extinction effect in discrete-trial lever pressing. Journal of Experimental Psychology: Animal Behavior Processes, 4(2), 131143. http://dx.doi.org/10.1037/0097-7403.4.2.131

Schoenfeld, W. N., Cumming, W. W., \& Hearst, E. (1956). On the classification of reinforcement schedules. Proceedings of the National Academy of Sciences, 42, 563-570. doi: 10.1073/ pnas.42.8.563

Sidman, M. (1960). Tactics of scientific research: Evaluating experimental data in psychology. New York, NY: Basic Books.

Siegel, P. S., \& Foshee, J. G. (1953). The law of primary reinforcement in children. Journal of Experimental Psychology, 45(1), 12-14. http://dx.doi.org/10.1037/h0054535

Thompson, T., Heistad, G. T., Palermo, D. S. (1963). Effect of amount of training on rate and duration of responding during extinction. Journal of Experimental Analysis of Behavior, 6(2), 155-161. http://dx.doi.org/10.1901/jeab.1963.6-155

Tombaugh, T. N., \& Jean, P. S. (1972). Overtraining extinction effect using a free-operant procedure. Psychological Records, 31, 539-544. http://dx.doi.org/10.2466/pr0.1972.31.2.539

Traupmann, K., \& Porter, J. J. (1971). The overlearning-extinction effect in free-operant bar pressing. Learning and Motivation, 2, 296-301. https://doi.org/10.1016/0023-9690(71)90030-0

Uhl, C. N. (1973). Eliminating behavior with omission and extinction after varying amounts of training. Animal Learning and Behavior, 1(3), 237-240. http://dx.doi.org/10.3758/BF03199082

Wanchisen, B. A. (1990). Forgetting the lessons of history. The Behavior Analyst, 13(1), 31-37. doi:10.1007/BF03392515

Weiner, H. (1965). Conditioning history and maladaptive human operant behavior. Psychological Reports, 17(3), 935-942. http:// dx.doi.org/10.2466/pr0.1965.17.3.935

Weiner, H. (1969). Controlling human fixed-interval performance. Journal of the Experimental Analysis of Behavior, 12(3), 349373. http://dx.doi.org/10.1901/jeab.1969.12-349

Weiner, H. (1970). Human behavioral persistence. Psychological Record, 20(4), 445-456. http://dx.doi.org/10.1007/BF03393965

Weiner, H. (1982). Histories of response omission and human operant behavior under a fixed-ratio schedule of reinforcement. Psychological Record, 32(3), 409-434. http://dx.doi. org/10.1007/BF03394799 